\title{
Influência da gramatura na resistência da linha de cola aos esforços de cisalhamento em painéis compensados de Copaifera duckei Dawyer e Eperua oleifera Ducke
}

\author{
Nerci Nina LIMA${ }^{1}$, Nabor da Silveira $\mathrm{PIO}^{2}$, Ulisses Silva da CUNHA ${ }^{3}$, Fernando Cardoso LUCAS FILHO \\ José BARBOSA FILHO5
}

\begin{abstract}
RESUMO
A madeira por ser um material de origem orgânica responde de várias maneiras aos diversos produtos nela empregados, inclusive a aplicação de cola a qual depende do seu teor de umidade, tendendo a atingir um teor de umidade em equilíbrio dinâmico com a umidade relativa da atmosfera. Atualmente utilizam-se lâminas com teores de umidade entre 5 e $15 \%$ de acordo com o tipo de resina utilizada. O presente estudo avaliou o efeito de 04 gramaturas $\left(270 ; 330 ; 364\right.$ e $\left.390 \mathrm{~g} / \mathrm{m}^{2}\right)$ na produçấo de painéis compensados utilizando as espécies: Copaifera duckei Dawyer e Eperua oleifera Ducke. As espécies utilizadas foram retiradas da área de manejo florestal sustentável da Gethal Indústria de Madeira Compensada Ltda localizada em ManicoréAm. As lâminas foram produzidas com espessuras de $2,2 \mathrm{~mm}$. As variáveis do ciclo de prensagem foram controladas seguindo as orientaçóes técnicas estabelecidas pelo fabricante da resina. Foi avaliada a resistência da linha de cola, com amostras da capa e do miolo em condiçôes seca e pós-fervura. Para análise estatística foi aplicado um delineamento inteiramente casualizado com arranjo em esquema fatorial dos tratamentos. Confrontando os valores médios da tensão de ruptura e a percentagem de falha na madeira obtidos nesta pesquisa, ensaio seco e pós-fervura, com os da literatura, foi verificado que os painéis avaliados atendem aos critérios estabelecidos, podendo ser indicados para uso interior e exterior, pois de acordo com a Norma EN 314-2 (1993) o valor médio da tensão de ruptura em conjunto com a porcentagem de falha na madeira encontram-se em um padrão aceitável de comportamento.
\end{abstract}

PALAVRAS-CHAVE: lâminas, linha de cola, adesivos.

\section{Effects of amount of adhesive spread on the glue line shear strength in the plywood of Copaifera duckei Dawyer and Eperua oleifera Ducke}

\begin{abstract}
The wood as a source of organic material responds in different ways to different treatments her employees, including the application of glue which depends on its moisture content, tending to reach a moisture content in dynamic equilibrium with relative humidity of the atmosphere. Currently we use blades with moisture levels of between 5 and $15 \%$ according to the type of resin used. This study evaluated the effect of 04 weights $(270,330,364$ and $390 \mathrm{~g} / \mathrm{m} 2)$ in the production of panels using offset the species: Copaifera ducker Dawyer and Eperua oleifera Ducke. The species were removed from the area of sustainable forest management Gethal Plywood Industry Ltd. located in Manicoré-Am. The slides were produced with thickness of 2.2 $\mathrm{mm}$. The variables of the pressing cycle of were controlled by following the technical guidelines set by the manufacturer of resin. The property was assessed to resilience of the line of glue, with samples of the hood and the kernels in drought conditions and post-boil. Statistical analysis was applied an experimental design with in a factorial arrangement of treatments. Confronting the average values of voltage collapse and the percentage of failure in wood obtained in this research, test and post-boiling dry, with the literature, it appears that the panels assessed meet the established criteria, may be indicated for use inside and outside Because according to EN 314-2 (1993) the average voltage collapse along with the percentage of failure in the wood are at an acceptable standard of behavior.
\end{abstract}

KEYWORDS: veneer, glue line, adhesive.

\footnotetext{
1 Universidade Federal do Amazonas.E-mail: nlima@ufam.edu.br

2 Universidade Federal do Amazonas - UFAM, Departamento de Ciências Florestais. E-MAIL: nspio@ufam.edu.br

3 Universidade Federal do Amazonas. E-mail: ulisses@ufam.edu.br

${ }^{4}$ Universidade Federal do Amazonas. E-mail: fecarlu@ufam.edu.br

${ }^{5}$ Universidade Federal do Amazonas. E-mail: jbarbosa@ufam.edu.br
} 


\section{INTRODUÇÃO}

Como a floresta Amazônica é rica em diversidade madeireira, várias espécies apresentam propriedades adequadas para usos finais como móveis, construçóes leves e pesadas, embalagens, pallets, pisos e chapas de compensado.

Barbosa et al (2001), cita que há centenas de espécies madeireiras que ocorrem na região, mas que ainda são desconhecidas ou pouco conhecidas, certamente existem várias com propriedades similares aquelas já tradicionais e de grande aceitaçáo para o mercado.

Com o avanço da tecnologia o setor produtivo de madeira da regiáo Norte vem sendo importante para a economia brasileira, pois com a estabilização da economia, o setor florestal passou a ser atrativo principalmente no mercado moveleiro (PIO, 2002).

Isto torna necessário promover nos mercados espécies regionais alternativas para substituir aquelas já tradicionalmente conhecidas. Tais espécies não são ainda comercializadas por absoluto desconhecimento de suas propriedades e potencial econômico.

A madeira por ser um material de origem orgânica responde de várias maneiras aos diversos produtos nela empregados, inclusive a aplicação de cola a qual depende do seu teor de umidade, tendendo a atingir um teor de umidade em equilíbrio dinâmico com a umidade relativa da atmosfera (KOLLMANN e COTE, 1968).

A reduçáo do teor de umidade da madeira envolve gastos de energia pelo processo de secagem. Esse processo e importante para produção de painéis compensados, que exige teores de umidade específicos com o objetivo de assegurar uma boa colagem. Atualmente utilizam-se lâminas com teores de umidade entre 5 e $15 \%$ de acordo com o tipo de resina utilizada (SILVA et alli, 1998).

$\mathrm{Na}$ colagem de lâminas de madeira para produção de compensados, diversos fatores devem ser considerados como as características inerentes a madeira, ao adesivo e procedimentos empregados na colagem (IWAKIRI et alli, 1999).

Devem-se verificar as conseqüências das determinadas alteraçôes qualitativas e quantitativas dos adesivos bem como as condiçóes de prensagem que podem influenciar na qualidade do produto final. $\mathrm{O}$ excesso de umidade com temperaturas de prensagem elevadas provocam falhas pela formação de bolhas debilitando a colagem (SELLERS, 1985).

O tempo de assemblagem é necessário para que haja compatibilidade da cola com a lâmina de madeira, antes de serem prensadas.

O processo de colagem é complexo, pois devem ser consideradas variáveis como temperatura, tempo de prensagem e pressão que associadas as características das pecas apresentam diferenças mesmo quando retiradas da mesma arvore. A colagem dependerá das características do adesivo, e de variáveis relacionada com o ambiente, com a estrutura morfológica da madeira, com a maneira que a madeira foi processada antes de ser colada, com a geometria da peça, etc (MARRA, 1992).

A influência da quantidade de cola na fabricaçấo de compensados em geral, é um fator importante, e está relacionada com o teor de umidade influindo decisivamente na resistência da junta e na estabilidade do produto colado (SELBO,1975).

Dependendo da viscosidade do adesivo, madeiras que possuem poros grandes absorvem mais cola conferindo melhor adesão. No entanto a gramatura deverá ser compensada sempre que houver alteração de espessura, pois lâminas mal torneadas (corrugadas) necessitam maior gramatura (IWAKIRI, 1998).

A linha de cola só apresentará problemas podendo gerar falhas no produto final se durante o processo de colagem não forem levados em consideraçóes fatores como a qualidade e viscosidade do adesivo, teor de umidade das lâminas e o grau de limpeza da superfície. Devido aos problemas encontrados na linha de cola pelas indústrias em geral, o custo elevado da matéria-prima empregado para compor o adesivo, bem como o processo técnico de aplicação, baseado normalmente em uma determinada quantidade $\mathrm{g} / \mathrm{m}^{2}$ de área, e necessário que se desenvolvam novas técnicas para otimizar o processo industrial, garantindo a qualidade do produto.

O presente artigo tem como objetivo avaliar o efeito de 04 gramaturas de cola $\left(270 ; 330 ; 364\right.$ e $\left.390 \mathrm{~g} / \mathrm{m}^{2}\right)$ na produção de painéis compensados de Copaiba mari-mari (Copaifera duckei Dawyer) e Copaíba jacaré (Eperua oleifera Ducke), através da propriedade de cisalhamento.

\section{MATERIAL E MÉTODOS}

\section{Amostragem e coleta do material}

As espécies utilizadas no presente estudo foram retiradas da área de manejo florestal sustentável da Gethal Indústria de Madeira Compensada Ltda localizada em Manicoré, no estado do Amazonas.

Foram utilizadas duas espécies florestais: Copaifera duckei Dawyer, (copaíba mari-mari), família Caesalpiniaceae e Eperua oleifera Ducke, (copaíba jacaré), família Caesalpiniaceae, sendo utilizada cinco arvores por espécie.

A decisão de trabalhar com essas espécies foi em virtude da disponibilidade do material (lâminas) pela empresa e por ainda serem poucos conhecidas quanto as suas propriedades fisico-mecanicas, quando utilizadas em painéis compensados principalmente a espécie Eperua oleifera que e a mais utilizada para este fim no estado do Amazonas. 
Após o abate, as toras foram transportadas por balsa para a Indústria de

Madeira Compensada Gethal Ltda, localizada no município de Itacoatiara - AM, onde foi realizado todo o processo de laminação das toras e fabricação dos painéis.

No pátio da empresa, as árvores foram traçadas seguindo as dimensóes padrão do torno lâminador da empresa de aproximadamente $2,65 \mathrm{~m}$. De cada árvore foram geradas 3 toras num total de 30 toras, sendo 15 da espécie Copaifera duckei e 15 de Eperua oleifera.

As espécies foram identificadas no laboratório de anatomia da madeira do CPPF - Coordenaçáo de Pesquisa de Produtos Florestais - INPA, através do método de comparação.

Os corpos-de-prova foram confeccionados na marcenaria do CPPF - INPA.

\section{Determinação da densidade básica da madeira}

Durante o traçamento que originou as toras para laminação, foram retirados 4 discos com $5 \mathrm{~cm}$ de espessuras da base, meio e topo da árvore.

Os discos de madeira foram utilizados para determinar a densidade básica das espécies.

\section{Manufatura dos painéis compensados}

Após o cozimento das toras, a uma temperatura em torno de $80^{\circ} \mathrm{C}$ por um período de $72 \mathrm{~h}$ as toras receberam um novo destopo, e em seguida foram desenroladas em um torno industrial automático. $\mathrm{O}$ torno foi aferido de acordo com o padrão da empresa para proporcionar uniformidade na espessura desejada.

As lâminas foram produzidas com espessuras de $2,2 \mathrm{~mm}$, aferidas por microton, seguindo o padrão da empresa. Ao saírem do torno, as lâminas foram enroladas em bobinas para em seguida serem guilhotinadas.

As lâminas foram secas em um secador industrial continuo a uma temperatura de $120^{\circ} \mathrm{C}$ por um período que variou entre 25 e 30 minutos com teor de umidade médio final de $6 \%$. O teor de umidade das lâminas após a secagem foi obtido através de medidor elétrico do tipo resistência.

Depois de secas, as lâminas foram classificadas em duas categorias: a de melhor qualidade para capa; e as inferiores para miolo. Essa classificação foi baseada na Norma de Controle de Qualidade e Classificação de Compensados (IBDF, 1985).

Cada chapa foi composta de nove lâminas com dimensóes de 2,55 x 1,22 x 0,0022 m. Foram produzidos 40 (quarenta) painéis, sendo 20 por espécie distribuídas em 8 tratamentos.

$\mathrm{Na}$ colagem das lâminas foi utilizado um adesivo a base de fenolformaldeido. A composição do adesivo foi basicamente da mistura simples da resina em uma batedeira com as demais partes componentes da formulaçáo adotada pela empresa, conforme tabela 1 .

Tabela 1 - Formulação do adesivo fenol-formoldeideo

\begin{tabular}{lccc}
\hline Adesivo & Componentes & Partes por peso & $(\%)$ \\
\hline & Resina FF & 108 & 75,52 \\
Fenol & Farinha de trigo & 15 & 10,49 \\
formaldeído & Água & 20 & 13,99 \\
Total & - & 143 & 100 \\
\hline
\end{tabular}

O controle das propriedades do adesivo após seu preparo foi realizado através do copo Ford para leitura da viscosidade, e para a medição do $\mathrm{pH}$ foi utilizado um Peagametro, verificando assim o estado e as condiçóes para utilização.

Foram utilizadas quatro diferentes gramaturas de cola aplicadas em linha dupla: 270, 330, $364 \mathrm{e} 390 \mathrm{~g} / \mathrm{m}^{2}$, valores normalmente utilizados pelas indústrias de compensados. A gramatura $364 \mathrm{~g} / \mathrm{m}^{2}$ e a utilizada pela empresa e foi tomada como testemunha.

O intervalo entre um tratamento e outro variou de 10 a 15 minutos, e a montagem de cada painel teve em media de 4,6 minutos para espécie Copaifera duckei e 3,8 minutos para espécie Eperua oleifera, com o tempo total de assemblagem de 5 horas, seguindo as recomendaçóes do fabricante da resina.

As variáveis do ciclo de prensagem foram controladas seguindo as orientaçóes técnicas estabelecidas pelo fabricante da resina, conforme tabela 2 abaixo.

Tabela 2 - Variáveis do ciclo de prensagem

\begin{tabular}{lc}
\hline Variáveis & Valores \\
\hline Adesivo & Resina fenol-formaldeido \\
Temperatura & $135^{\circ} \mathrm{C}$ \\
Tempo de prensagem & $20 \mathrm{~min}$. \\
Gramatura da cola & $270 ; 330 ; 364$ e $390 \mathrm{~g} / \mathrm{m}^{2}$ \\
Pressão especifica & $12 \mathrm{~kg} / \mathrm{cm}^{2}$ \\
Umidade da lâmina & $6 \%$ \\
\hline
\end{tabular}

\section{Avaliação dos painéis}

Apos a prensagem dos painéis, os mesmos foram transportados para a sala de climatizaçáo no laboratório de física da madeira da Universidade Federal do Amazonas, e acondicionados a uma temperatura de $20^{\circ} \mathrm{C}$ até entrarem em equilíbrio com o ambiente, o teor de umidade foi verificado aleatoriamente através de medidor elétrico.

O principal fator avaliado nos painéis foi o comportamento das diferentes gramaturas: $270 ; 330 ; 364 \mathrm{e} 390 \mathrm{~g} / \mathrm{m}^{2}$ ao esforço de cisalhamento, para determinar sua propriedade. 
Foram adotadas as normas Européias EN 310 e 314-2 (1993) que descrevem procedimentos para os ensaios de resistência da linha de cola e flexão estática.

Os corpos de prova para os ensaios mecânicos foram retirados a $5 \mathrm{~cm}$ da borda dos painéis, evitando o efeito da bordadura, e as dimensôes dos corpos-de-prova foram: $25 \mathrm{~mm}$ x $800 \mathrm{~mm}$ x espessura painel.

Os testes mecânicos foram realizados no laboratório de tecnologia da madeira do curso de engenharia florestal da Universidade Federal do Paraná.

O ensaio de resistência da linha de cola foi realizado com amostras da capa, onde era testada a resistência da linha de cola da lâmina da capa do painel, e lâminas do miolo onde era testada a resistência da linha de cola da lâmina do miolo, em condiçóes seco corpos-de-prova a umidade ambiente e pós-fervura, corpos-de-prova com alto grau de stress. O número de corpos de prova para cada espécie e ensaio é mostrado na tabela 3.

Tabela 3 - Números de corpos de prova para ensaio por espécie e normas utilizadas

\begin{tabular}{|c|c|c|c|c|c|}
\hline Espécie & Ensaio & Condição & $\begin{array}{l}\text { Corpo de } \\
\text { prova }(p / p)\end{array}$ & $\begin{array}{l}\text { Total } \\
\text { cps }\end{array}$ & Norma \\
\hline $\begin{array}{l}\text { Copaifera } \\
\text { duckei }\end{array}$ & Cisalhamento & $\begin{array}{c}\text { Seco } \\
\text { Pós-fervura }\end{array}$ & $\begin{array}{l}10 \\
10\end{array}$ & $\begin{array}{l}200 \\
200\end{array}$ & EN310:1993 \\
\hline $\begin{array}{l}\text { Eperua } \\
\text { oleifera }\end{array}$ & Cisalhamento & $\begin{array}{c}\text { Seco } \\
\text { Pós-fervura }\end{array}$ & $\begin{array}{l}10 \\
10\end{array}$ & $\begin{array}{l}200 \\
200\end{array}$ & EN310:1993 \\
\hline
\end{tabular}

Nota: $(p / p)=$ corpo de prova por painel

Cps: corpos de prova

\section{Análise estatística}

Foi aplicado um delineamento inteiramente casualizado com arranjo em esquema fatorial dos tratamentos utilizando o programa estatístico SISVAR conforme Tabela 4.

Tabela 4 - Espécies, variáveis e gramaturas aplicadas nos tratamentos

\begin{tabular}{|c|c|c|c|c|}
\hline Espécie & Ensaio & $\begin{array}{l}\text { Variáveis } \\
\text { Seco e Pós- } \\
\text { fervura }\end{array}$ & *Tratamentos & $\begin{array}{c}\text { Gramatura } \\
\left(\mathrm{g} / \mathrm{m}^{2}\right)\end{array}$ \\
\hline \multirow{4}{*}{ Copaifera duckei } & \multirow{4}{*}{ Cisalhamento } & \multirow{4}{*}{$\begin{array}{l}\text { Capa e } \\
\text { miolo }\end{array}$} & $\mathrm{T} 1$ & 270 \\
\hline & & & T2 & 330 \\
\hline & & & T3 & 364 \\
\hline & & & T4 & 390 \\
\hline \multirow{4}{*}{ Eperua oleifera } & \multirow{4}{*}{ Cisalhamento } & \multirow{4}{*}{$\begin{array}{c}\text { Capa e } \\
\text { miolo }\end{array}$} & $\mathrm{T} 1$ & 270 \\
\hline & & & T2 & 330 \\
\hline & & & T3 & 364 \\
\hline & & & T4 & 390 \\
\hline
\end{tabular}

*Para cada tratamento foram usadas cinco repetições

A tabela 5 mostra o esquema da análise de variância realizado.
Tabela 5 - Analise de Variância da interação gramatura x espécie

\begin{tabular}{lc}
\hline Fator de Variação & Graus de Liberdade \\
\hline Gramatura & 3 \\
Espécie & 1 \\
Gramatura x espécie & 3 \\
Erro & 28 \\
\hline Total & 39 \\
\hline
\end{tabular}

\section{RESULTADOS E DISCUSSÃO}

\section{Densidade básica}

A densidade básica e importante para a determinação da temperatura ótima de cozimento da tora e do tempo de aquecimento necessário para temperaturas desejadas no cerne, (KEINERT JUNIOR, 1994).

A tabela 6 mostra os valores médios da densidade básica, desvio padrão e coeficiente de variação por espécie.

Tabela 6 - Valores médios da densidade, desvio padrão e coeficiente de variação

\begin{tabular}{lccc}
\hline Espécie & $\begin{array}{r}\text { Densidade básica } \\
\left(\mathrm{g} / \mathrm{cm}^{3}\right)\end{array}$ & Desvio padrão & $\begin{array}{c}\text { Coeficiente de } \\
\text { variação }(\%)\end{array}$ \\
\hline $\begin{array}{l}\text { Copaifera } \\
\text { duckei }\end{array}$ & 0,60 & 0,0858 & 14,40 \\
$\begin{array}{l}\text { Eperua } \\
\text { oleifera }\end{array}$ & 0,62 & 0,0681 & 10,97 \\
\hline
\end{tabular}

De acordo com a Tabela 6 há pouca diferença entre as espécies estudadas e segundo Lutz (1978), espécies com valores de densidade básica variando entre 0,40 e $0,70 \mathrm{~g} / \mathrm{cm}^{3}$ são adequadas para produção de lâminas.

O valor da densidade básica para a espécie Copaifera duckei $\left(0,60 \mathrm{~g} / \mathrm{cm}^{3}\right)$, foi próximo ao valor $0,62 \mathrm{~g} / \mathrm{cm}^{3}$ encontrado por IBAMA (2001).

\section{Propriedades dos painéis}

\section{Resistência da linha de cola - ensaio miolo e capa seco}

Este ensaio tem como objetivo determinar o grau de colagem em chapas compensadas e avaliar a resistência do painel. Por meio do valor obtido é possível avaliar a porcentagem de falha na madeira complementando o resultado do ensaio.

Os valores obtidos devem avaliar as condiçóes físicas e mecânicas do uso das chapas antes e após o seu envelhecimento. Este ensaio é efetuado sob duas condiçóes: teste de cisalhamento a seco e teste de cisalhamento com fervura dos corpos de prova o que provoca situaçấo de elevado stress ao produto.

A análise de variância realizada, para o ensaio de resistência da linha de cola e falha na madeira miolo seco, para as espécies 
estudadas indicam não haver diferenças significativas, a nível de $5 \%$ de probabilidade, entre os tratamentos avaliados.

Tabela 7 - Valores médios de resistência da linha de cola (rlc) e falha na madeira

\begin{tabular}{lcccc}
\hline Gramatura de & \multicolumn{2}{c}{ RLC Miolo seco $\left(\mathrm{N} / \mathrm{mm}^{2}\right)$} & \multicolumn{2}{c}{ FNM Miolo seco $(\%)$} \\
\cline { 2 - 5 } Cola $\left(\mathrm{g} / \mathrm{cm}^{2}\right)$ & C. duckei & E. oleifera & C. duckei & E. oleifera \\
\hline (T1) 270 & $2,97^{\mathrm{ns}}$ & $2,87^{\mathrm{ns}}$ & $38,2^{\mathrm{ns}}$ & $29,7^{\mathrm{ns}}$ \\
(T2) 330 & $3,88^{\mathrm{ns}}$ & $3,10^{\mathrm{ns}}$ & $34,4^{\mathrm{ns}}$ & $31,4^{\mathrm{ns}}$ \\
(T3) 364 & $3,66^{\mathrm{ns}}$ & $3,45^{\mathrm{ns}}$ & $20,4^{\mathrm{ns}}$ & $15,0^{\mathrm{ns}}$ \\
(T4) 390 & $3,67^{\mathrm{ns}}$ & $2,93^{\mathrm{ns}}$ & $17,0^{\mathrm{ns}}$ & $46,0^{\mathrm{ns}}$ \\
\hline
\end{tabular}

(FNM) para a posicao miolo seco

ns- Tratamento que não diferem da testemunha ao nível de 5\%

A Tabela 7 apresenta os valores médios de resistência da linha de cola e falha na madeira para os ensaios de miolo seco.

Entretanto, entre as espécies, houve diferença significativa para posiçáo miolo seco. Os dados estáo apresentados na Tabela 8. Isso indica que os tratamentos analisados para Copaifera duckei obteve o melhor resultado de maneira que a interação entre cola e madeira e maior para esta espécie.

Tabela 8 - Valores médios de rlc para a variável resposta espécie posição miolo seco

\begin{tabular}{lcc}
\hline Espécie & Número de painéis & $\mathrm{RLC}\left(\mathrm{N} / \mathrm{mm}^{2}\right)$ Miolo seco \\
\hline Copaifera duckei & 20 & $3,55^{\mathrm{ns}}$ \\
Eperua oleifera & 20 & 3,09 * \\
\hline
\end{tabular}

RLC: Resistência da Linha de Cola

ns-Não significativo

*- Significativo ao nível de $5 \%$ pelo Teste de Tukey

A tabela 8 mostra os valores médios para a RLC no miolo ensaio a seco.

O resultado da análise de variância do ensaio capa seco da resistência de linha de cola e falha na madeira, indicam diferença estatística significativa para a espécie Eperua oleifera a $5 \%$ de probabilidade. Os valores médios são apresentados na Tabela 9.

Tabela 9 - Valores médios de resistência da linha de cola (rlc) e falha na madeira (fnm) para a posição capa seca - espécies copaifera duckei e eeperua oleifera

\begin{tabular}{lcccc}
\hline Gramatura de & \multicolumn{2}{c}{ RLC Capa seco $\left(\mathrm{N} / \mathrm{mm}^{2}\right)$} & \multicolumn{2}{c}{ FNM Capa seco $(\%)$} \\
\cline { 2 - 5 } Cola $\left(\mathrm{g} / \mathrm{cm}^{2}\right)$ & C. duckei & E. oleifera & C. duckei & E. oleifera \\
\hline (T1) 270 & $2,73^{\text {ns }}$ & $2,80^{*}$ & $99,2^{\text {ns }}$ & $93,4^{\text {ns }}$ \\
(T2) 330 & $2,96^{\text {ns }}$ & $3,12^{*}$ & $95,0^{\text {ns }}$ & $96,3^{\text {ns }}$ \\
(T3) 364 & $3,05^{\text {ns }}$ & $2,28^{*}$ & $98,6^{\text {ns }}$ & $95,6^{\text {ns }}$ \\
(T4) 390 & $2,87^{\text {ns }}$ & $2,84^{*}$ & $93,6^{\text {ns }}$ & $98,8^{\text {ns }}$ \\
\hline
\end{tabular}

Teste Unilateral de Dunnett

* - Tratamento que diferem da testemunha ao nível de 5\%

ns - Tratamento que não diferem da testemunha

Não houve diferenças significativas para a resistência ao cisalhamento posição capa seco da espécie Copaifera duckei, porém, na interação dos fatores estudados, espécie Eperua oleifera, foi verificado diferenças significativas. Os tratamentos analisados pelo Teste Dunnett, todos foram superiores ao T3 (controle), indicando que para esta espécie as gramaturas analisadas são inferiores a testemunha.

Quanto à falha na madeira também não apresentaram diferença significativas, a nível $5 \%$ de probabilidade, para as espécies estudadas.

\section{Resistência da linha de cola - ensaio miolo e capa pós-fervura}

Para o ensaio de resistência da linha de cola posição miolo pós-fervura e falha na madeira, os resultados da análise de variância para as espécies Copaifera duckei e Eperua oleifera, não apresentaram diferenças significativas entre os tratamentos.

A Tabela 10 apresenta os valores médios de resistência da linha de cola e falha na madeira para as espécies estudadas.

Com relação aos valores do teste de média para resistência da linha de cola e falha na madeira ensaio posição capa pósfervura, os resultados da análise de variância para as espécies Copaifera duckei e Eperua oleifera indicam que não houve diferença significativa.

A Tabela 11 revela os valores médios para os ensaios de resistência da linha de cola e falha na madeira posição capa pós-fervura.

Tabela 10 - Valores médios de resistência da linha de cola (rlc) e falha na madeira

\begin{tabular}{lcccc}
\hline \multirow{2}{*}{$\begin{array}{l}\text { Gramatura de } \\
\text { cola }\left(\mathrm{g} / \mathrm{cm}^{2}\right)\end{array}$} & \multicolumn{2}{c}{$\begin{array}{c}\text { RLC Miolo pós-fervura } \\
\left(\mathrm{N} / \mathrm{mm}^{2}\right)\end{array}$} & \multicolumn{2}{c}{ FNM Miolo pós-fervura (\%) } \\
\cline { 2 - 5 } & C. duckei & E. oleifera & C. duckei & E. oleifera \\
\hline (T1) 270 & $1,52^{\mathrm{ns}}$ & $1,67^{\mathrm{ns}}$ & $11,0^{\mathrm{ns}}$ & $48,6^{\mathrm{ns}}$ \\
(T2) 330 & $1,49^{\mathrm{ns}}$ & $1,68^{\mathrm{ns}}$ & $14,6^{\mathrm{ns}}$ & $49,0^{\mathrm{ns}}$ \\
(T3) 364 & $2,03^{\mathrm{ns}}$ & $1,98^{\mathrm{ns}}$ & $13,6^{\mathrm{ns}}$ & $44,8^{\mathrm{ns}}$ \\
(T4) 390 & $2,15^{\mathrm{ns}}$ & $1,65^{\mathrm{ns}}$ & $15,8^{\mathrm{ns}}$ & $47,0^{\text {ns }}$ \\
\hline
\end{tabular}

(FNM) para posição I miolo pós-fervura - espécie Copaifera duckei e Eperua oleifera ns- Tratamento que não diferem da testemunha ao nivel de $5 \%$ Fonte: Dados da Pesquisa

Tabela 11 - Valores médios de resistência da linha de cola (RLC) e falha na madeira

\begin{tabular}{lcccc}
\hline $\begin{array}{l}\text { Gramatura } \\
\text { de cola }\end{array}$ & $\begin{array}{c}\text { RLC } \\
\text { Miolo Pos-Fervura } \\
\left(\mathrm{N} / \mathrm{mm}^{2}\right)\end{array}$ & \multicolumn{2}{c}{$\begin{array}{c}\text { MNM } \\
\text { Miolo Pos-Fervura } \\
(\%)\end{array}$} \\
\hline (g/m²) & C. duckei & E. oleifera & C. duckei & E. oleifera \\
(T1) 270 & $1,70^{\text {ns }}$ & $1,59^{\text {ns }}$ & $93,8^{\text {ns }}$ & $99,8^{\text {ns }}$ \\
(T2) 330 & $1,81^{\text {ns }}$ & $1,73^{\text {ns }}$ & $95,8^{\text {ns }}$ & $96,0^{\text {ns }}$ \\
(T3) 364 & $1,80^{\text {ns }}$ & $1,47^{\text {ns }}$ & $97,0^{\text {ns }}$ & $98,4^{\text {ns }}$ \\
(T4) 390 & $1,63^{\text {ns }}$ & $1,56^{\text {ns }}$ & $90,8^{\text {ns }}$ & $95,4^{\text {ns }}$ \\
\hline
\end{tabular}

(FNM) para posição capa pós-fervura - espécies Copaifera duckei e Eperua oleifera ns - Tratamento que não diferem da testemunha ao nível de 5\% 
A Tabela 12 apresenta a comparaçáo dos resultados de resistência de linha de cola e falha na madeira obtidos neste trabalho com outros da literatura.

De acordo com Pio (1996) e Silva (1994) o teste de cisalhamento é o que determina a qualidade da linha de cola. Comparando os valores obtidos nesta pesquisa em relação a gramatura de cola com os valores encontrados na Tabela 13, observa-se que apesar da diferença entre a gramatura de cola existente nesta pesquisa com os autores citados, a resistência da linha de cola ensaio seco para as duas espécies apresentaram comportamento consideravelmente superior.

Quanto à percentagem de falha na madeira apresentaram resultados superiores em relação à capa tanto para o ensaio seco como pós-fervura em comparação aos demais autores. Porém esses autores utilizaram a norma (ABNT, 1986) o qual náo especificava nenhum valor para tensão de ruptura e falha na madeira, pois a mesma estava em processo de revisão.

Portanto, confrontando os valores médios da tensão de ruptura e a percentagem de falha na madeira obtidos nesta pesquisa, ensaio seco e pós-fervura, com os valores médios apresentados na Tabela 13, foram verificados que os painéis avaliados atendem aos critérios estabelecidos, podendo ser indicados para uso interior e exterior, pois de acordo com a Norma EN 314-2 (1993) o valor médio da tensão de ruptura em conjunto com a porcentagem de falha na madeira proporciona uma avaliaçáo mais completa e adequada sobre a qualidade da colagem o que náo devem ser menores que o limite estabelecido conforme tabela abaixo.

Assim, com base na Tabela 13, conforme aumenta a tensão de ruptura a exigência em termos de falha na madeira reduz, ate o momento em que pode ser desprezada, ou seja, quando a tensão de ruptura for muito alta (igual ou superior a 10,2 $\mathrm{kgf} / \mathrm{cm}^{2}$ ou $\left.1,0 \mathrm{Mpa}\right)$.

Considerando os valores obtidos nesta pesquisa, os quais foram superiores em todos os tratamentos e com base nos requisitos da norma européia, os compensados avaliados podem ser indicados para uso exterior.

Tabela 12 - comparação dos resultados de resistência da linha de cola obtidos com outras literaturas

\begin{tabular}{|c|c|c|c|c|c|c|c|}
\hline \multirow{2}{*}{ Fonte } & \multirow{2}{*}{ Espécie } & \multirow{2}{*}{ Espes Lâm. (mm) } & \multirow{2}{*}{ Gram. $\left(\mathrm{g} / \mathrm{cm}^{2}\right) \mathrm{FF}$} & \multicolumn{2}{|c|}{ FNM (\%) } & \multicolumn{2}{|c|}{$\mathrm{RLC}\left(\mathrm{kgf} / \mathrm{cm}^{2}\right)$} \\
\hline & & & & Seca Miolo/cap & Pós-ferv.. & Seca Miolo/cap & Pós-ferv. \\
\hline Gaiotto 1993 & E. grandis & 2 & 180 & 78 & 98 & 20,54 & 15,56 \\
\hline Bortoletto 2003 & Eucalyptus & 2 & 360 & $0-34,3$ & $0,6-17,2$ & $22,6-27,4$ & $19,1-24,2$ \\
\hline Medina 1986 & P. elliottii & 3 & 300 & 80 & 54 & 2,49 & - \\
\hline Pio 1996 & $\begin{array}{l}\text { E. robusta } \\
\text { E. scabra }\end{array}$ & 2 & 180 & $\begin{array}{l}34 \\
48 \\
\end{array}$ & - & $\begin{array}{l}21,86 \\
25,98 \\
\end{array}$ & - \\
\hline Interamnense & $\begin{array}{l}\text { E. cloziana } \\
\text { E. maculata }\end{array}$ & 2 & 175 & $\begin{array}{l}42 \\
37 \\
\end{array}$ & $\begin{array}{l}40 \\
35 \\
\end{array}$ & $\begin{array}{l}21,24 \\
25,28 \\
\end{array}$ & $10,8-16,6$ \\
\hline \multirow{8}{*}{ Dissertação 2006} & \multirow{4}{*}{ C. duckei } & \multirow{4}{*}{2,2} & 270 & $38,2-99,2$ & $11,0-93,8$ & $30,3-27,8$ & $15,5-17,3$ \\
\hline & & & 330 & $34,4-95,0$ & $14,6-95,8$ & $39,5-30,2$ & $15,2-18,4$ \\
\hline & & & 364 & $20,4-98,6$ & $13,6-97,0$ & $37,3-31,1$ & $20,7-18,3$ \\
\hline & & & 390 & $17,0-93,6$ & $15,8-90,8$ & $37,4-29,3$ & $21,9-16,6$ \\
\hline & \multirow{4}{*}{ E. oleifera } & \multirow{4}{*}{2,2} & 270 & $29,7-93,4$ & $48,6-99,8$ & $29,3-28,5$ & $17,0-16,2$ \\
\hline & & & 330 & $31,4-96,3$ & $49,0-96,0$ & $31,6-31,8$ & $17,1-17,6$ \\
\hline & & & 364 & $15,0-95,6$ & $44,8-98,4$ & $35,1-23,2$ & $20,1-15,0$ \\
\hline & & & 390 & $46,2-98,8$ & $47,0-95,4$ & $29,8-28,9$ & $16,8-15,9$ \\
\hline
\end{tabular}

Nota:* Os valores de resistência de linha de cola foram transformados para $\mathrm{kgf} / \mathrm{cm}^{2}$ para serem comparados

Tabela 13 - requisitos de colagem para painéis compensados

\begin{tabular}{cc}
\hline \multicolumn{2}{c}{ Resistência da linha de cola AP esforço de cisalhamento } \\
\hline Tensão de ruptura $\left(\mathrm{N} / \mathrm{mm}^{2}\right)$ & Ruptura ou Falha na Madeira (\%) \\
\hline $0,2 \leq \mathrm{TR}<0,4$ & $\geq 80$ \\
$0,4 \leq \mathrm{TR}<0,6$ & $\geq 60$ \\
$0,6 \leq \mathrm{TR}<1,0$ & $\geq 40$ \\
$1,0 \leq \mathrm{TR}$ & Sem exigências \\
\hline
\end{tabular}

Fonte: EN 314-2 (1993)

\section{CONCLUSÕES}

Os resultados obtidos nos ensaios de resistência de linha de cola quando comparados com as especificaçōes da norma EN 314-2 (1993), indicam que os painéis das duas espécies avaliadas podem ser destinados ao uso exterior. 
Para os painéis onde os tratamentos não indicaram diferenças significativas entre as gramaturas avaliadas, tanto para a posição capa quanto miolo, pode-se indicar a menor gramatura de cola.

A gramatura de melhor desempenho foi a $330 \mathrm{~g} /$ $\mathrm{m}^{2}$ quando avaliada na posição capa para a espécie Copaíba jacaré (Eeperua oleifera.)

Comparando os valores obtidos nesta pesquisa com os valores encontrados na literatura, observa-se que a gramatura de cola utilizada nesta pesquisa foi similar para a posição capa ensaio seco dos demais autores indicando boa qualidade da linha de cola dos painéis.

A porcentagem de ruptura ou falha na madeira apresentou valores superiores, porém, compatíveis com outros autores.

\section{BIBLIOGRAFIA CITADA}

BARBOSA, A.P, et al. Consideraçôes sobre o perfil tecnológico do setor madeireiro na Amazônia Central: In biodiversidade, pesquisa e desenvolvimento na Amazônia. Parcerias Estratégicas. no.12. Setembro 2001. 421 p.

BORTOLETTO JUNIOR, G. Produção de compensados com 11 espécies do gênero Eucalyptus, avaliação de suas propriedades físico-mecânicas e indicaçôes para utilização. Revista Scientia Forestalis, n. 63, p. 65-78, jun. 2003.

EUROPEAN COMMITTEE FOR STANDARDIZATION. European Standard EN - 310/314-2. Plywood: Bonding quality. Part 2: Requirements. Bruxelas. 1993.

GAIOTTO, M.R.; WATAI, L.T.; JANKOWSKY, I.P. Avaliação experimental do compensado de eucalipto. In: Congresso Florestal Panamericano e 7o Congresso Florestal Brasileiro, Anais. Curitiba. 1993. 595-599 p.

IBAMA - Instituto Brasileiro do Meio Ambiente. Madeiras Brasileiras. Disponível em: <http://www.ibama.gov.br/lpf/ madeira/caracteristicas.php > Acesso em: 21 janeiro 2001.

IBDF - Instituto Brasileiro de Desenvolvimento Florestal. Normas de Controle de Qualidade e Classificação de Compensados-CQCC. Brasília. $1985.79 \mathrm{p}$.

INTERAMNENSE, M.T. Utilização das madeiras de Eucalyptus Cloeziana (F.MUELL), Eucalyptus maculata (Hook) e Eucalyptus puncata DC var. puncata para produção de painéis compensados. 1998. 82 f. Dissertação (Mestrado em Tecnologia de Produtos
Florestais). Curso de Pós-Graduação em Engenharia Florestal. Universidade Federal do Paraná, Curitiba.

IWAKIRI, S; et al. Produção de chapas de madeira aglomerada de Pinus elliottii Engelm com inclusão laminar. Cerne. V.5, n.2, Lavras. 1999. 94p.

KEINERT JUNIOR, S. Laminação, Produção e Testes de Compensados a partir de Eucalyptus spp. Relatório de Pesquisa apresentado ao CNPq. (não publicado).Curitiba. 1994. 93 p.

KOLLMANN, F. F. P.; COTE, W. A. Principles of wood science and technology. New York: Springer-Verlag, v.1. 1968.

KOLLMANN, F.F.P.; KUENZI, E.W.; STAMM, A.J. Principles of wood science and technology. v. 2 - Wood based materials. Berlin, New York .1975. 703 p. LUTZ, J.F. Woody veneer: log selection, cutting and drying. USDA. Technical Bulletin. 1978.

MARRA, A. A. Technology of wood bonding principles in practice. New York: Van Nostrand Reinhold. 1992. 453 p.

MEDINA, J. C. Efeito da temperatura de aquecimento na obtenção de lâminas por desenrolamento e sobre a qualidade da colagem de compensados fenólicos de Pinus elliottii Engelm. 1986. 113 f. Dissertação (Mestrado em 73 Tecnologia e Utilização de Produtos Florestais). Universidade Federal do Paraná, Curitiba.

PIO, N. S. Avaliação da madeira de Eucalyptus scabra (Dum-Cours) e Eucalyptus robusta (Smith) na produção de painéis compensados. 1996. 101 f. Dissertação (Mestrado em Tecnologia e Utilização de Produtos Florestais). Curso de Pós-Graduaçáo em Engenharia Florestal, Universidade Federal do Paraná, Curitiba.

PIO, N. S. Produção de painéis estruturais de lâminas paralelas (PLP) de Eucalyptus grandis Hill ex-Maiden. 2002. 168 f. Tese (Doutorado em Ciências Florestais). Curso de Pós-Graduação em Engenharia Florestal, Universidade Federal do Paraná, Curitiba.

SELBO, M. L. Adhesive Bonding of wood. Technical Bulletin. $\mathrm{n}^{\circ}$ 1512. U. S. Department of Agriculture. Forest Service. Washington D.C. 1975.

SELLERS, T. Plywood and adhesive technology. New York: Marcel Dekker. 1985. 661 p.

SILVA, D.A.; TOMASELLI, I.; IWAKIRI, S. Influência da umidade na resistência da linha de cola e estabilidade dimensional do compensado utilizando resina de alta reatividade. SCIENTIA FORESTALIS. n 54, p.69-80, dez. 1998.

SILVA, F.M. Espécies tropicais para manufatura de painéis compensados. 1994. 68 f. Dissertação (Mestrado em Recursos Florestais). Escola Superior de Agricultura Luiz de Queiroz. Piracicaba, SP.

Recebido em 27/02/2009

Aceite em 06/04/2010 
